\title{
Exploring the effects of a non-interactive talking avatar on social presence, credibility, trust, and patronage intention in an e-commerce website
}

\author{
Tze Wei Liew ${ }^{1}$, Su-Mae Tan ${ }^{2 *}$ and Hishamuddin Ismail ${ }^{1}$
}

\section{*Correspondence:}

smtan@mmu.edu.my

${ }^{2}$ Multimedia University, Jalan Ayer Keroh Lama,

75450 Melaka, Malaysia Full list of author information is available at the end of the article

\begin{abstract}
While prior research has examined the effects of interactive e-commerce avatars that simulate the roles of virtual assistants and recommender agents, there is lack of empirical study that investigates the effects of non-interactive talking avatars in e-commerce. This is unfortunate, as many websites today utilize non-interactive talking avatars that provide one-way dialogue in the forms of greetings, introduction of the company, and description of products and services offered. To bridge this gap, our study aims to investigate the effects of non-interactive talking avatar on perceptions of social presence, credibility, website trust, and patronage intention in an online store. Comparing between the experimental (website with avatar) and control (website without avatar) conditions, the experimental results showed that perceived social presence was significantly enhanced by the presence of the non-interactive talking avatar. Furthermore, the presence of the avatar had positive effects on website trust and patronage intention among male participants; but had negative effects on perceptions of information credibility and website patronage intention among females. Analysis based on participants' comments suggests that females were more critical towards certain shortcomings of the non-interactive talking avatar; citing limited interactivity and artificial dialogue from text-to-speech engine. Additionally, the comment analysis suggests that females preferred processing textual over auditory information (as spoken by the avatar) about the products in the online store. Theoretical and managerial implications related to non-interactive talking avatars are discussed in this paper.
\end{abstract}

Keywords: Talking avatar, Interactivity, Social presence, Information credibility, Website trusts, Patronage intention, Gender, E-commerce

\section{Introduction}

The process of shopping is fundamentally social. The lack of social stimulus in e-commerce websites is one of the key barriers to positive consumer attitudes towards online shopping $[1,2]$. Indeed, e-commerce sites often emphasize more about being proficient and effective, hence neglect the "human touch". In the endeavour to infuse social cues in online stores, researchers have incorporated platforms such as live chats, forums, and social media [2]. Additionally, human images, custom greetings, and videos of human

(c) The Author(s) 2017. This article is distributed under the terms of the Creative Commons Attribution 4.0 International License (http://creativecommons.org/licenses/by/4.0/), which permits unrestricted use, distribution, and reproduction in any medium, provided you give appropriate credit to the original author(s) and the source, provide a link to the Creative Commons license, and indicate if changes were made. 
beings can be integrated in website design to increase the sense of social presence in online stores [3]. It has also been suggested that the integration of talking avatars in online stores can help resolve the lack of social cues in e-commerce sites [4-7].

Avatars can be defined as "virtual characters that can be used as company representatives" in online stores [8]. They are endowed with animation, video, audio, and text-tospeech technologies, to convey verbal and nonverbal cues to users. Recent years have seen the nascent integration of talking avatars in e-commerce sites. For instance, the website of BobDog Wine features a talking dog avatar that conveys greetings and information about the company. The website of Qarbon Solutions integrates a talking avatar in a form of a photorealistic human that greets and briefly introduces the company products. Katie and Kimble blog uses a child character as talking avatar that delivers welcome message and information regarding new products.

The psychological effects of talking avatars on consumer behaviours can be ascribed to the theory of Computers as Social Actors (CASA) [9]. According to the CASA theory, people will unconsciously apply social rules to computers, especially when the computers exhibit human-like attributes. Hence, social cues such as facial expressions and conversational dialogues from talking avatars can trigger automatic social responses in people. For instance, some of the social rules found relevant in human-computer interaction include stereotype [10], inhibition/facilitation [11], politeness [12], personality matching/mismatching [13, 14], empathy [15], emotional contagion [16, 17], specialistgeneralist attribution [18, 19], and venting [20]. Likewise, consumers tend to psychologically profile e-commerce avatars as (1) virtual assistant, (2) friendly host, and (3) recommender agent [21]. Avatars as virtual assistants provide help to consumers by pointing out special offers, guiding them through the payment process, pointing out sources of information, and searching the Web for requested items. Avatars, as friendly hosts, deliver welcome messages, farewell messages, and personalized greetings. They also provide guided tours of sites and description of products and services. Avatars as recommender agents provide suggestions of items based on personal consumer profile and past purchases.

In recent years, research studies have examined the effects of presence (against the absence) of avatars on consumer behaviours in e-commerce websites (see Table 1 for a review). In most of these studies, the embodied avatars took the roles of virtual assistants and recommender agents. The interactivity capabilities afforded by these avatars were able to simulate two-way conversational dialogue by allowing users to give input during a communication, usually via text, and then provide instantaneous (real-time) spoken or textual responses. The presence of interactive avatars has been shown to enhance perceptions of social presence $[4,5,22]$, credibility [22], website trusts $[4,5,22$, $23]$, and website patronage intention $[4,5]$.

However, many establishments today are employing non-interactive talking avatars that simulate the roles of welcome hosts in their websites, e.g., BobDog Wine, Qarbon Solutions, EGlobal Studies, OnCue Productions, Technology Solutions Forall, and DNA Boots. These avatars simply provide one-way conversational dialogue that is usually generated through text-to-speech computer voices, to greet users, introduce the company, and describe the products and services offered. While non-interactive avatars are found in many websites, research has yet to empirically examine the effects of non-interactive 
Table 1 Summary of studies comparing the effects of the presence versus absence of e-commerce avatars

\begin{tabular}{|c|c|c|c|}
\hline Author & Role of avatar & Interactivity of avatar & Findings \\
\hline Alves and Soares [22] & $\begin{array}{l}\text { Describe the product } \\
\text { information using verbal } \\
\text { dialogue }\end{array}$ & No & $\begin{array}{l}\text { Avatar with real voice had the } \\
\text { highest effects on trust, cred- } \\
\text { ibility, sociability, and human } \\
\text { warmth. Avatar with text-to- } \\
\text { speech did not significantly } \\
\text { affect trust and credibility } \\
\text { as compared to non-avatar } \\
\text { condition }\end{array}$ \\
\hline Holzwarth [8] & $\begin{array}{l}\text { Dispense advice and recom- } \\
\text { mendation related to prod- } \\
\text { uct (helper role/personal } \\
\text { shopper/recommender } \\
\text { agent) }\end{array}$ & Yes & $\begin{array}{l}\text { Avatars led to more satisfac- } \\
\text { tion with the retailer, a more } \\
\text { positive attitude towards } \\
\text { the product, and a greater } \\
\text { purchase intention. Attractive } \\
\text { avatars were more persua- } \\
\text { sive when consumers were } \\
\text { moderately involved in the } \\
\text { purchase whereas expert } \\
\text { avatars were marginally more } \\
\text { persuasive when consumers } \\
\text { were highly involved in the } \\
\text { purchase }\end{array}$ \\
\hline Qiu and Benbasat [6] & $\begin{array}{l}\text { Dispense advice and recom- } \\
\text { mendation related to prod- } \\
\text { uct (helper role/personal } \\
\text { shopper/recommender } \\
\text { agent) }\end{array}$ & Yes & $\begin{array}{l}\text { The presence of avatar did not } \\
\text { significantly influence trust } \\
\text { among consumers. Avatars } \\
\text { with text-to-speech induced } \\
\text { higher cognitive trust in con- } \\
\text { sumers than avatars with text }\end{array}$ \\
\hline Etemad-Sajadi [23] & $\begin{array}{l}\text { Helper role/personal shop- } \\
\text { per/recommender agent }\end{array}$ & Yes & $\begin{array}{l}\text { Avatar's social presence had a } \\
\text { significant impact on trust } \\
\text { and emotional appeal, which } \\
\text { led to increased online real- } \\
\text { time interaction }\end{array}$ \\
\hline Chattaraman et al. [5] & $\begin{array}{l}\text { Guide users through the } \\
\text { shopping tasks (helper role) }\end{array}$ & Yes & $\begin{array}{l}\text { Avatar presence enhanced } \\
\text { perceived interactivity, social } \\
\text { support, anxiety alleviation, } \\
\text { trust, and intention to revisit } \\
\text { as compared to a visually } \\
\text { absent avatar among elderly } \\
\text { users }\end{array}$ \\
\hline Chattaraman et al. [4] & $\begin{array}{l}\text { Guide users through the } \\
\text { shopping tasks (helper role) }\end{array}$ & Yes & $\begin{array}{l}\text { Avatar presence enhanced } \\
\text { patronage intention, social } \\
\text { support, website trust (ability, } \\
\text { benevolence, and integrity) } \\
\text { and reduced perceived risk as } \\
\text { compared to a visually absent } \\
\text { avatar among elderly users }\end{array}$ \\
\hline
\end{tabular}

talking avatars on consumer behaviours. Indeed, we note that non-interactive avatars are currently underrepresented in research. Furthermore, the advantages of interactive avatars as reflected in the previous works should not be generalized to non-interactive avatars. This is due to the fact that interactivity, which is one of the fundamental qualities of avatars simulating virtual assistants and recommender agents, is an inherent factor that lends its impact in influencing social presence, credibility, website trust, and patronage intention. It has been argued that "online shoppers are more likely to accept and use an agent if it affords a compelling, socially rich and enjoyable interaction experience, in addition to product search and comparison functionalities" [24]. Indeed, Etemad-Sajadi 
[23] notes that real-time interactivity afforded by interactive avatars serves both hedonistic and utilitarian purposes. Specifically, hedonic value focuses on fun, playfulness, and emotional worth; while utilitarian value focuses on the efficiency to reach the desired information on the website. Hence, there is a chasm that divides the research findings related to interactive avatars and non-interactive avatars featured in many websites today. Whether or not non-interactive talking avatars can induce higher social presence, credibility, website trust, and patronage intention remains an open question. Hence, this paper aims at bridging the research-practice gap.

The present study also aims to investigate if consumer gender moderates the effects of non-interactive talking avatars on perceptions of social presence, credibility, website trust, and patronage intention. The moderating factor of user gender has been a neglected area of research related to conversational avatars. Based on the scant literature related to this domain, it was found that user gender moderated the effects of avatar designs on consumer behaviours $[6,19]$, and that females are generally less trusting towards information dispensed by e-commerce agents as compared to males [25]. In adddition, it has been suggested that female online shoppers benefit more from the induced social presence by avatars than their male counterparts [21]. These aforementioned findings provide a reasonable cause for this paper to examine the moderating effects of consumer gender in the effects of non-interactive talking avatars on consumer behaviours. Therefore, the secondary objective of this study is to explore the interaction effect between user gender and the presence of a non-interactive talking avatar on perceived social presence, information credibility, website trust, and patronage intention.

\section{Theoretical background}

\section{Talking avatar and social presence}

Social presence is characterized as the capacity of a medium to allow the user to sense the environment as, "warm", "personal", and "sociable" [1, 2, 26]. The concept of social presence is closely related to the media richness theory which defines vividness and interactivity as social stimuli in the communication medium $[1,2]$. Vividness refers to the extent to which a medium produces stimulus that allows consumers to experience sensory input, while interactivity refers to characteristics of the channel that simulate face-to-face interaction [27]. Leaning on this premise, it can be surmised that online stores generally have lower degrees of social presence than conventional brick-andmortar stores. Unlike physical stores that provide opportunities for sensory experiences (browse, touch, and feel products) and social relationships (interaction with sales advocates and peer shoppers), online stores are mostly anonymous and automated platforms, with little opportunity for social contact.

To resolve this issue, it has been suggested that embedding verbal and nonverbal stimuli such as facial expressions, vocal tones, eye gazes, and gestures can heighten perceptions of social presence in technologically-mediated channels [28]. Adhering to this point, researchers have attempted to enrich online stores with social presence by implementing live chats, personalized greetings, human photos, virtual communities, and discussion boards [3, 29]. Going a step further, talking avatars offer the advantage of being able to simultaneously and seamlessly deliver both verbal and nonverbal communication (facial expressions, gestures, voice), thus imbuing online stores with vivid and interactive 
elements [27]. In an avatar-implemented website, the combination of visual and audio cues by the talking avatar allows users to experience richer sensory stimuli and interactivity, hence increases perceived social presence as "warm", "personal", and "sociable".

Moreover, as per the Computers as Social Actors theory [9], consumers reciprocate avatars' interactions by forming social connections with them. Specifically, the social agency theory [30] posits that social cues in multimedia communication can prime social conversation schema in users. When social conversation schema is activated, automatic social response impels users to assign social connotations to the interaction. Besides, the persona effect indicates that the presence of human pictures is sufficient to create a sense of social presence in users [31]. Building on the aforementioned discussions, talking avatars can increase the sense of social presence by realistically simulating the role of sales advocates in online stores. In the traditional sales environment, perceptions of social presence are strongly dependent on interaction with sales advocates. Without face-to-face interaction, shopping experience becomes impersonal, cold, and distant. In this regard, talking avatars can mimic "frontline" advocates to provide social cues such as welcome messages, farewell messages, personalized greetings, and guided tour on products and services.

Empirical studies have shown evidence supporting the facilitating effects of talking avatars on social presence. Qiu and Benbasat [6] demonstrated that consumers who interacted with recommender agents with human faces and voices, experienced a higher sense of social presence than did consumers who interacted with a text-based recommender agent. In a study by Choi et al. [27], it was found that a web advertisement delivered by a talking avatar induced higher experience of social presence in consumers than a text-based web advertisement. The effects of conversational avatar on social presence was also observed in 3D virtual stores in which consumers had higher perceptions of social presence when talking avatars representing the salesperson and peer shopper were present, than when avatars were absent [32]. The study by Alves and Soares [22] revealed that online stores with talking avatars (experimental) induced higher perceptions of social presence in consumers, than a text-based e-commerce website (control). Finally, Chattaraman et al. $[5,6]$ showed that the implementation of conversational avatars increased perceived social presence in senior-aged consumers.

However, the issue of non-interaction concerning non-interactive talking avatars requires further scrutiny. Arguably, avatar interactivity that simulates two-way conversation, is one of the key variables in the aforementioned social presence frameworks such as social agency and media richness. For instance, two-way dialogue is necessary to activate social conversation schema in user's mind. Furthermore, interactivity allows virtual avatars to better simulate their roles as customer service representatives. Prior research experiments examining the social presence effects of avatars have used embodied agents that have interactive capabilities, i.e., able to provide responses to user inputs (see Table 1). Although many websites today utilizes non-interactive talking avatars to provide one-way greeting and product information, research is relatively silent on the effectiveness of such type of avatars on social presence. Indeed, whether or not the heightened social presence induced by interactive avatars may be replicated with noninteractive talking avatars (which lack the elements of interactivity) remains an open question. Therefore, there is a need for empirical research to examine the effectiveness of 
non-interactive talking avatars in inducing social presence in e-commerce sites, thereby giving rise to the following question:

RQ 1: How does the presence (as against the absence) of a non-interactive talking avatar affect perceptions of social presence in an online store?

\section{Talking avatar and information credibility}

Information credibility is a crucial factor in e-commerce. Credibility can be described as the extent to which the source and its message are perceived to be believable [22]. The psychology judgement of credibility of information on websites are influenced by the source or messenger. As noted by Koh and Sundar [33], consumers tend to automatically assign responsibility of the messages to those who deliver them and feel that the most proximate messenger is the source. Therefore, talking avatars are considered the source messengers when delivering product description to consumers via pre-recorded human voices or text-to-speech dialogues.

In an experiment by Alves and Soares [22], it was found that the presence of avatar with pre-recorded human voice enhanced credibility in users when compared to control (no avatar) condition. However, it was also shown that avatar with text-to-speech voice did not significantly enhance credibility when compared with the control condition. Therefore, one may infer that text-to-speech computer voices may be ineffective in inducing higher sense of credibility among online users. Prior literature has also pointed out that design factors of avatars affect perceived information credibility [31]. Notwithstanding, there are limited studies that examine the effects of talking avatar presence on information credibility. In an attempt to bridge this research gap, we present the following research question:

RQ 2: How does the presence (as against the absence) of a non-interactive talking avatar affect perceptions of information credibility in an online store?

\section{Talking avatar and website trusts}

Trust can be described as "a psychological state comprising the intention to accept vulnerability based upon positive expectations of the intentions or behavior of another" [34]. Generally speaking, consumers must trust online retailers not to exploit the websites through unscrupulous strategies such as unfair pricing, violations of privacy, and security or misrepresentation of information. However, due to the lack of development and enforcement of rules and regulations protecting consumers' rights, online stores face challenges in gaining trust. In the context of e-commerce, the trust construct has often been conceptualized as beliefs in vendor's ability, benevolence, and integrity [35].

- Ability is defined as the capabilities, skills, and knowledge of a vendor that allows the seller to successfully perform actions related to the business domain.

- Benevolence is defined as the sincerity of the vendor in placing priority on the best interest of consumers, in contrast to a mere profit motive.

- Integrity refers to the vendor's tendency to honour agreements in good faith, truthful representation of information, ethical business conduct, and fulfilment of promises. 
Integrity consists of the characteristics of sellers such as honesty, fulfilment, fairness, loyalty, and dependability.

Talking avatars, with their capability of infusing social cues in e-commerce sites, have been shown to enhance website trusts in online shoppers [4-6]. From a theoretical perspective, the facilitating effects of conversational avatars on trust can be attributed to two reasons. First, it is instructive to note that trust is built upon familiarity [36]. The rich amalgam of verbal and nonverbal signals by avatars can produce a sense of familiarity in users, who are generally accustomed to sensory experiences acquired in conventional physical stores (e.g., interaction with sales advocates). In this regard, the social presence simulated by talking avatars meet the expectations of consumers to encounter "familiar human elements" when shopping online. Second, social cues such as body gestures, eye contact, facial expressions, and voice tones are crucial factors in trust formation. Resonating with this notion, the visual and auditory aspects of talking avatars can serve as "assurance" signals to users. In order to reduce social uncertainty in a new environment, people naturally seek peripheral cues that add to their sense of familiarity. Similarly, when consumers visit an online store, their first objective is to reduce uncertainty and ambiguity by searching for any peripheral cues. Social cues afforded by conversational avatars allow consumers to form social and psychological impressions of the technologically-mediated agent, thus reducing the perceived ambiguity regarding the intentions and expected behaviours of the medium and the e-tailer. With the reduction in uncertainty and ambiguity related to websites and vendors, trust is facilitated.

However, one may argue that non-interactive talking avatars may be ineffective in giving assurance to consumers, due to their limited functionalities in responding to user queries or inputs. In fact, such limitations may even reduce website trust (particularly trust-ability) because it projects the image that the website or e-tailer does not possess the capabilities, skills, and knowledge that allow the seller to successfully perform actions related to the business domain. Furthermore, perceptions of trust-benevolence and trust-integrity may suffer as well due to the one-way dialogue offered by non-interactive talking avatars. Indeed, monologues are not in line with social interaction norms, thereby leading to feelings of distrusts, uncertainty, and ambiguity. To assess this issue, we ask the following question:

RQ 3: How does the presence (as against the absence) of a non-interactive talking avatar affect perceptions of website trust [ability (RQ3a), benevolence (RQ3b), integrity (RQ3c)] in users?

\section{Talking avatar and website patronage intention}

Patronage intention of website refers to the likelihood of consumers revisiting the e-commerce in the future [37]. As per the Theory of Planned Behaviour [38], behavioural intentions are the most significant predictors of behaviour. Patronage intention is related to other behavioural indicators such as repeat purchases, repeat website visits, and endorsement of the website to fellow shoppers [39]. Thus, in the context of this study, patronage intention is used to measure purchase behaviours. Alves and Soares [22] showed that the integration of photorealistic avatars with human voices led to 
increased intentions to purchase and repurchase. Chattaraman et al. $[4,5]$ demonstrated that interactive virtual avatars increased website patronage intention among senior consumers. While past studies have shown that interactive conversational avatars increased website patronage intentions, we wonder if such effects can also be manifested in the context of non-interactive talking avatars. More formally,

RQ 4: How does the presence (as against the absence) of a non-interactive talking avatar affect website patronage intention in an online store?

\section{Consumer's gender as moderating effect}

In this research, the role of consumer's gender is explored. In accordance with prior literature $[3,6,40]$, female consumers are thought to be more receptive to social and hedonistic aspects of website interfaces, than their male counterparts. Dittmar et al. [40] notes that male consumers focus more on the utilitarian and functional aspects of shopping whereas, female consumers focus more on social-experiential, identity-related, and emotional involvements. The findings by Cyr et al. [3] supports this notion; it was shown that most of the open-ended comments by male consumers were related to utilitarian aspects of the website such as information adequacy and security features. In contrast, female consumers expressed opinions about the emotional and social tones of the website, such as, "friendliness", "vibrancy", and "curiosity".

Qiu and Benbasat [6] demonstrated that the ethnicity-match effects of conversational avatars on social presence and perceived enjoyment were more significant for female consumers than for their male counterparts. Moreover, McGoldrick et al. [21] predicted that females would be more approving of avatars as friendly hosts, due to a higher need for rapport. The hypothesis was revealed to be true as it was found that the female respondents appreciated the avatars, particularly their friendly roles, more than the male respondents. In terms of credibility and trusts, females are generally less trusting towards e-commerce agents than males [25]. This suggests that perceptions of information credibility and trusts related to talking avatars may be moderated by consumer gender. Furthermore, as females are more sensitive towards social cues delivered by anthropomorphic agents $[6,19]$; therefore, they may be more critical towards the limited interactivity of non-interactive talking avatars as compared to males. Taking into account all these possibilities, we ask:

RQ 5: How does the consumer gender moderate the effects of presence (as against the absence) of a non-interactive talking avatar on perceptions of social presence (RQ5a), information credibility (RQ5b), website trust (RQ5c), and patronage intention (RQ5d)?

\section{Methods}

\section{Experimental design}

The study employed a 2 (non-interactive talking agent presence: present versus absent) $\times 2$ (user gender: male versus female) between-subjects laboratory experiment design. 


\section{Participants}

We used convenience sampling where subjects were selected because of their convenient accessibility and proximity to the researcher. Hence, sophomores, $(n=116 ; 52$ males, 64 females; mean age $=19$ ) enrolled in the course "E-Commerce" at an Asian private university, were invited to participate in the experiment in exchange of partial course credits. Among the participants, the ethnicity composition was $60.34 \%$ Chinese, $34.48 \%$ Malay, and $5.17 \%$ Indian.

\section{Development of avatar and online store}

For the purpose of this experiment, a talking avatar, personified as Mina, was created. The visual and animation aspects of Mina were developed using Voki, a speaking avatar platform (http://www.voki.com). The speech of the avatar was developed using IVONA, a text-to-speech engine platform using the voice of Salli, English (http://www.ivona. com). Two versions of the multi-category product online store (control and experimental) were developed. In the control and experimental conditions, both websites offer a balanced mix of hair styling appliances, healthcare self-monitoring kits, camera equipment and washing machines. In the experimental website, the talking avatar (Mina) was embedded in all the webpages. On the homepage, Mina introduced herself as a sales agent, conveyed a welcome greeting to users, and delivered a brief description of the online store and its products. On the product webpages, supplementing the brief textual descriptions of the products, Mina delivered the full audio descriptions of products. The experimental website was also designed in such a way that when users exit the webpage of the online store, a short-duration pop-up box would appear with Mina conveying her thanks and farewell. The control website was identical in interface design, function, and

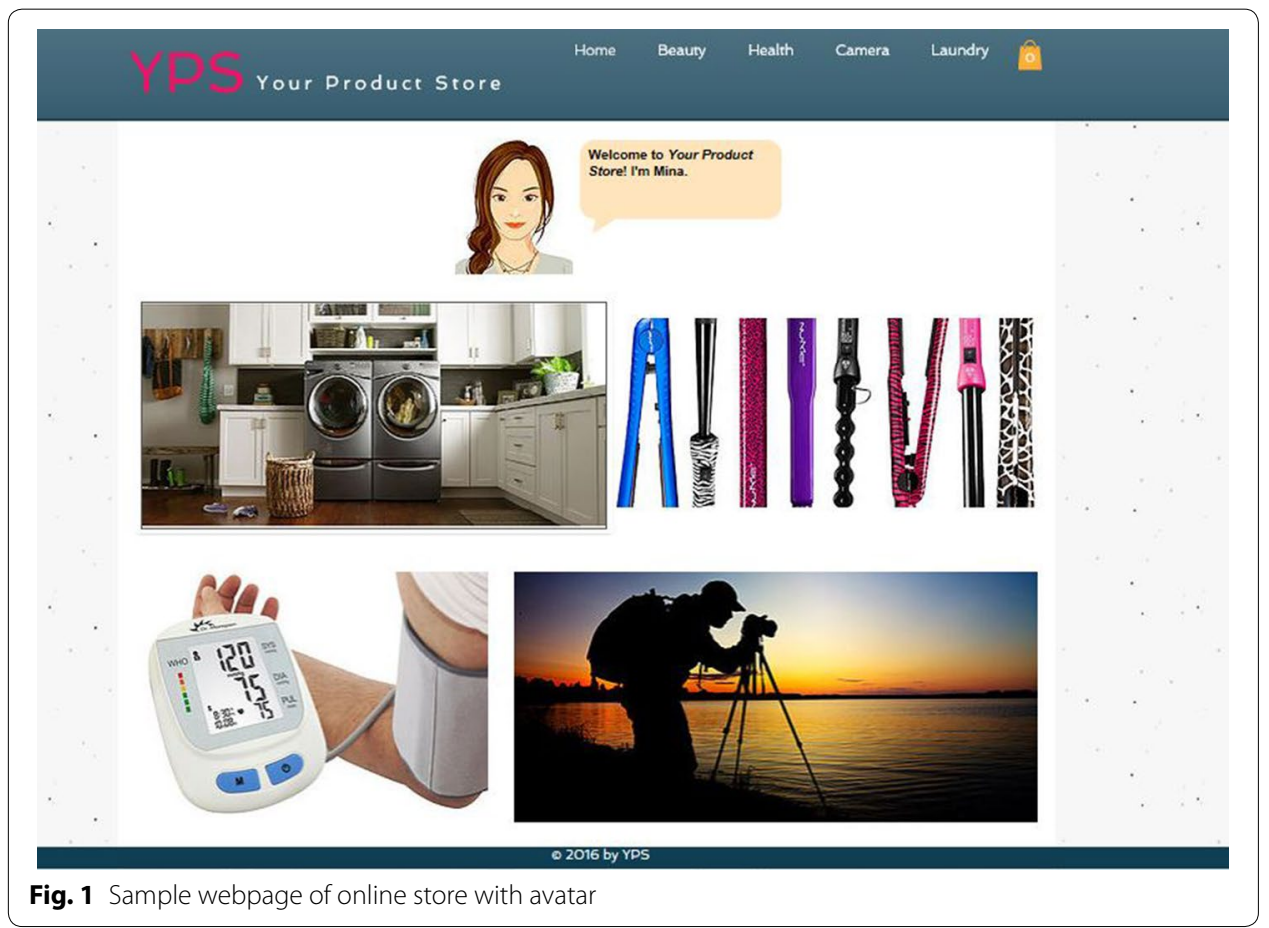




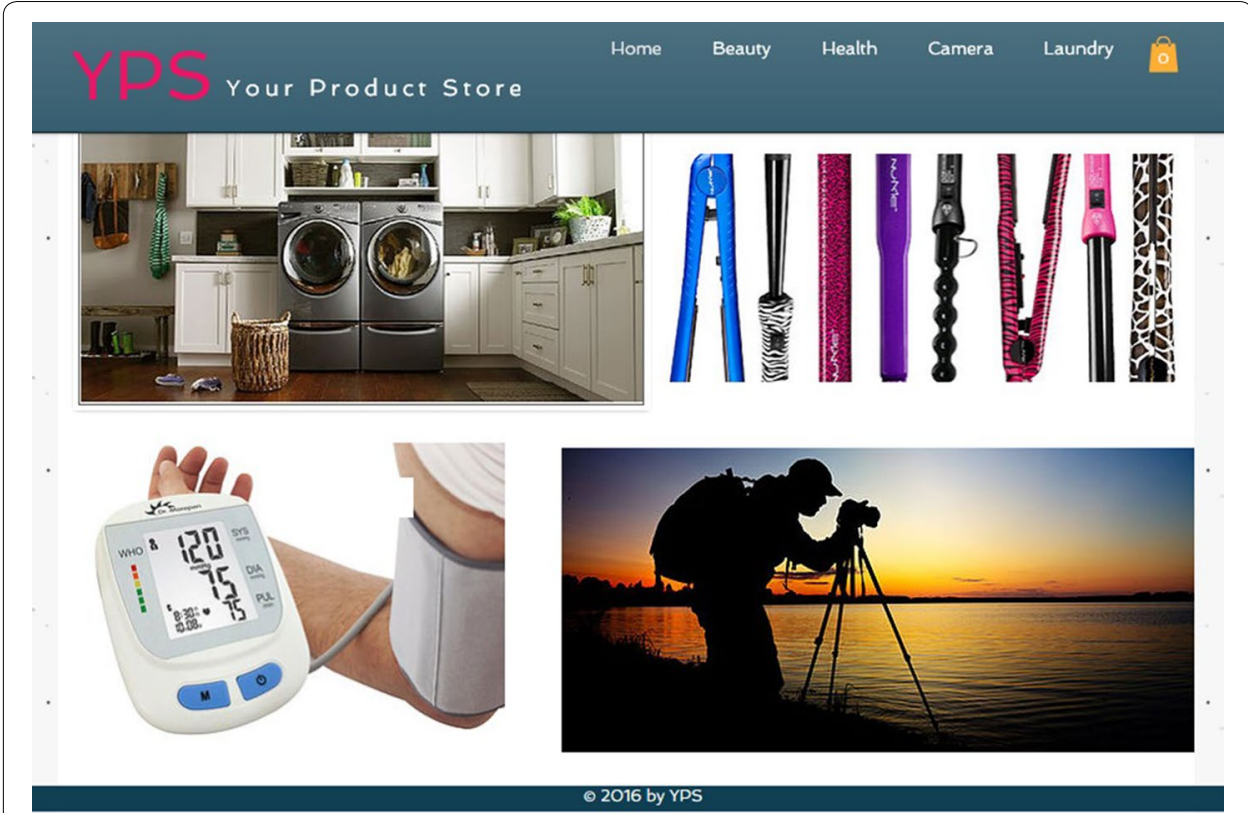

Fig. 2 Sample webpage of online store without avatar

content, except for the absence of Mina, the talking avatar. Figures 1 and 2 illustrate the websites of the experimental and control conditions.

\section{Dependent measures}

All measures in the questionnaire were graded on a 5-point Likert scale and adapted from established and validated measurement instruments. Table 2 shows the items of the dependent measures.

\section{Experimental procedure}

Participants were randomly divided and ushered into one of the two identically designed laboratories, thereby establishing the experimental (non-interactive talking avatar) and control (no avatar) groups. Seated in front of individual computers with headphones, participants of both groups were asked to navigate through the e-commerce website. They were required to add three products into the shopping cart and proceed to "check out" the items. After the interaction with their respective website, participants were instructed to complete the surveys via Google forms. Participants were dismissed after debriefing about the purpose of the study. The whole experimental procedure took approximately $1 \mathrm{~h}$.

\section{Results}

RQ 1: How does the presence (as against the absence) of a non-interactive talking avatar affect perceptions of social presence in an online store?

An independent-samples $t$ test was conducted to compare the average scores of perceived social presence between participants in the experimental (non-interactive talking avatar present) and control (non-interactive talking avatar absent) conditions. The results revealed that participants experienced significantly more social presence in the 
Table 2 Items of the dependent measures

\begin{tabular}{|c|c|c|}
\hline Construct & Items & Cronbach's alpha \\
\hline Perceived social presence [3] & $\begin{array}{l}\text { There is a sense of human contact in the website } \\
\text { There is a sense of personal ness in the website } \\
\text { There is a sense of sociability in the website } \\
\text { There is a sense of human warmth in the website } \\
\text { There is a sense of human sensitivity in the website }\end{array}$ & .921 \\
\hline Perceived information credibility [31] & $\begin{array}{l}\text { The information provided by the website is accurate } \\
\text { The information provided by the website is credible } \\
\text { The information provided by the website is factual }\end{array}$ & .892 \\
\hline Trust benevolence [35] & $\begin{array}{l}\text { I believe that this website would act in my best } \\
\text { interest } \\
\text { If I required help, this website would do its best to } \\
\text { provide assistance } \\
\text { This website is interested in my well-being and not } \\
\text { just its own }\end{array}$ & .909 \\
\hline Trust ability [35] & $\begin{array}{l}\text { This website is competent and effective in its interac- } \\
\text { tions with me } \\
\text { This website performs all of its roles very well } \\
\text { Overall, this website is capable and proficient } \\
\text { In general, this website is informative }\end{array}$ & .863 \\
\hline Trust integrity [35] & $\begin{array}{l}\text { This website is truthful in its dealing with me } \\
\text { I would characterize this website as being honest } \\
\text { This website keeps its commitments } \\
\text { This website is sincere and genuine }\end{array}$ & .915 \\
\hline Website patronage intention [37] & $\begin{array}{l}\text { I would visit this website again } \\
\text { I would shop on this website again } \\
\text { I would patronize this website }\end{array}$ & .981 \\
\hline
\end{tabular}

online store when the non-interactive talking avatar was present $(\mathrm{M}=3.72, \mathrm{SD}=.76)$, than when the non-interactive talking avatar was absent $(\mathrm{M}=3.32, \mathrm{SD}=1.04)$, $\mathrm{t}(114)=2.377, \mathrm{p}=.019$.

RQ 2: How does the presence (as against the absence) of a non-interactive talking avatar affect perceptions of information credibility in an online store?

Comparing the average survey scores for perceived information credibility, the independent-samples $t$ test revealed no significant difference between the non-interactive talking avatar present $(\mathrm{M}=3.55, \mathrm{SD}=.84)$ and non-interactive talking avatar absent $(\mathrm{M}=3.83, \mathrm{SD}=.81)$ conditions, $\mathrm{t}(114)=1.767, \mathrm{p}=.08$.

RQ 3: How does the presence (as against the absence) of a non-interactive talking avatar affect perceptions of website trust [ability (RQ3a), benevolence (RQ3b), integrity (RQ3c)] in users?

A series of independent-samples t tests was performed to compare the effects of presence versus the absence of a non-interactive talking avatar on website trust-ability, website trust-benevolence, and website trust-integrity. Based on the results, it was found that participants who interacted with online store that had a non-interactive talking avatar $(\mathrm{M}=4.05, \mathrm{SD}=.74)$ assigned significantly higher trust-benevolence than did participants who interacted with online store that had no non-interactive talking avatar $(\mathrm{M}=3.61, \mathrm{SD}=1.18), \mathrm{t}(114)=2.454, \mathrm{p}=.016$. In terms of trust-ability, there was no significant difference of average scores between the avatar present $(\mathrm{M}=4.65, \mathrm{SD}=.99)$ and avatar absent conditions $(\mathrm{M}=4.39, \mathrm{SD}=1.06), \mathrm{t}(114)=1.337, \mathrm{p}=.184$. The results 
also showed no significant difference of average scores with respect to trust-integrity between avatar-present $(\mathrm{M}=4.45, \mathrm{SD}=.78)$ and avatar-absent conditions $(\mathrm{M}=4.37$, $\mathrm{SD}=.91), \mathrm{t}(114)=.487, \mathrm{p}=.627$.

RQ 4: How does the presence (as against the absence) of a non-interactive talking avatar affect website patronage intention in an online store?

Results from the independent samples $t$ test showed that there was no significant difference between the non-interactive talking avatar present $(\mathrm{M}=2.86, \mathrm{SD}=.58)$ and noninteractive talking avatar absent $(\mathrm{M}=2.80, \mathrm{SD}=.69)$ conditions for website patronage intention, $\mathrm{t}(114)=.552, \mathrm{p}=.582$.

RQ 5: How does the user gender moderate the effects of presence (as against the absence) of a non-interactive talking avatar on perceptions of social presence (RQ5a), information credibility (RQ5b), website trusts (RQ5c), and patronage intention (RQ5d)?

A series of 2 (talking avatar presence: present versus absent) $\times 2$ (user gender: male versus female) ANOVAs was performed for perceived social presence, information credibility, and patronage intention; and a 2 (talking avatar presence: present versus absent) $\times 2$ (user gender: male versus female) MANOVA was conducted for website trust (ability, benevolence, integrity).

For perceived social presence, the results from the 2-way ANOVA showed a non-significant interaction effect between talking avatar presence and user gender $[F(1,112)=2.166, p=.144]$. In terms of perceived information credibility, the results revealed a significant interaction effect between talking avatar presence and user gender, $[\mathrm{F}(1,112)=7.293, \mathrm{p}=.008$, partial eta squared $=.061]$. Planned comparison $\mathrm{t}$ test (split by user gender) showed that the presence versus the absence of talking avatar did not significantly affect perceived information credibility among males, $\mathrm{t}(50)=.81, \mathrm{p}=.422$. However, it was found that females perceived the information in the experimental (talking avatar present; $\mathrm{M}=3.35, \mathrm{SD}=.79$ ) condition to be significantly less credible than the information in the control (talking avatar absent; $M=4.00, S D=.95$ ) condition, $\mathrm{t}(62)=3.153, \mathrm{p}=.002$.

For website trust (ability, benevolence, integrity), the 2-way MANOVA results showed significant interaction effects between talking avatar presence and user gender for trust-ability $[\mathrm{F}(1,112)=3.911, \mathrm{p}=.05$, partial eta squared $=.034]$ and trustintegrity $[\mathrm{F}(1,112)=7.289, \mathrm{p}=.008$, partial eta squared $=.061]$. Planned comparison $t$ tests (split by user gender) showed that the effects of talking avatar presence on website trust-ability and website trust-integrity were only significant among males, and not for females. Specifically, males assigned significantly higher trust-ability to the website that had a talking avatar $(\mathrm{M}=4.96, \mathrm{SD}=.67)$ than to the website that had no talking avatar $(\mathrm{M}=4.3, \mathrm{SD}=.71), \mathrm{t}(50)=3.392, \mathrm{p}=.001$. Furthermore, it was also found that males assigned significantly higher trust-integrity to the website that had a talking avatar $(\mathrm{M}=4.79, \mathrm{SD}=.73)$ than to the website that had no talking avatar $(\mathrm{M}=4.26$, $\mathrm{SD}=.73), \mathrm{t}(50)=2.474, \mathrm{p}=.017$.

In terms of website patronage intention, the results from the 2-way ANOVA showed that there was a significant interaction effect between talking avatar presence and user gender $[\mathrm{F}(1,112)=24.79, \mathrm{p}=.000$, partial eta squared $=.181]$. Based on the results 
from planned comparison $t$ test (split by user gender), it was found that the presence of the non-interactive talking avatar had a positive impact on patronage intention among males, as they assigned significantly higher ratings of patronage intention for the website that had a talking avatar $(\mathrm{M}=3.03, \mathrm{SD}=.53)$ than for the website that had no talking avatar $(\mathrm{M}=2.35, \mathrm{SD}=.50), \mathrm{t}(50)=4.596, \mathrm{p}=.000$. Contrariwise, females assigned significantly lesser ratings of patronage intention for the website that had a talking ava$\operatorname{tar}(\mathrm{M}=2.72, \mathrm{SD}=.59)$ than for the website that had no talking avatar $(\mathrm{M}=3.12$, $\mathrm{SD}=.62), \mathrm{t}(62)=2.615, \mathrm{p}=.011$.

Figures 3, 4, 5, and 6 illustrate the significant interaction effects of non-interactive talking avatar presence and user's gender on perceived information credibility, website trust-integrity, website trust-benevolence, and website patronage intention.

\section{Discussion}

\section{Theoretical implications}

The findings of this study have some theoretical implications. Previous studies examining the effects of embodied avatars on social presence utilized avatars with interactive capabilities, such that they are able to provide real-time responses to user inputs in order to mimic the roles of virtual assistants and recommender agents. Perceived realtime interactivity is arguably a key factor in inducing social presence among online consumers $[23,24,29]$. However, our study found preliminary evidence that perceived social presence can be heightened by the presence of non-interactive talking avatar in an online store. Notably, this finding emerged despite the fact that the talking avatar used in our experiment merely provided one-way spoken dialogue, i.e., deliver welcome message,

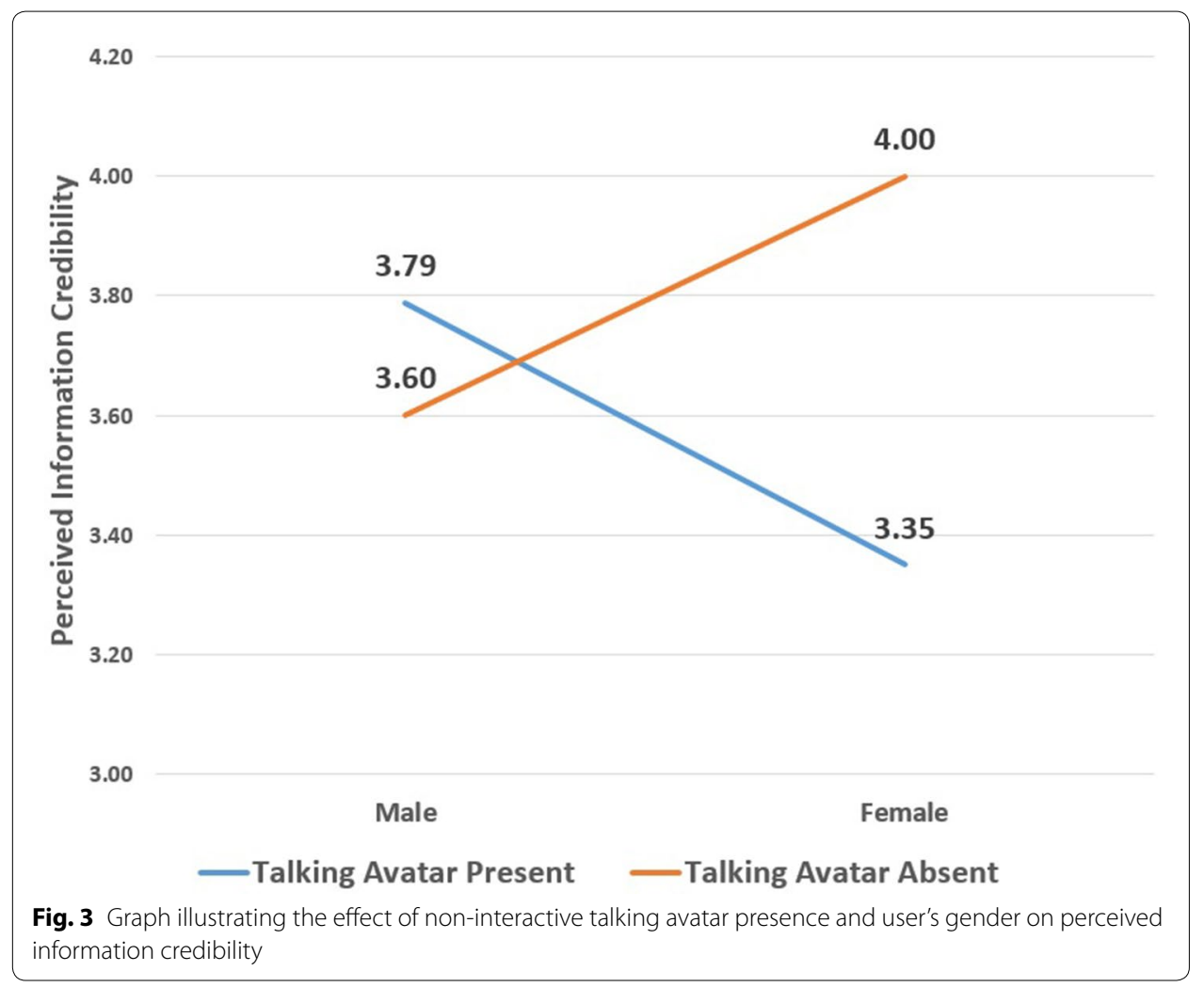




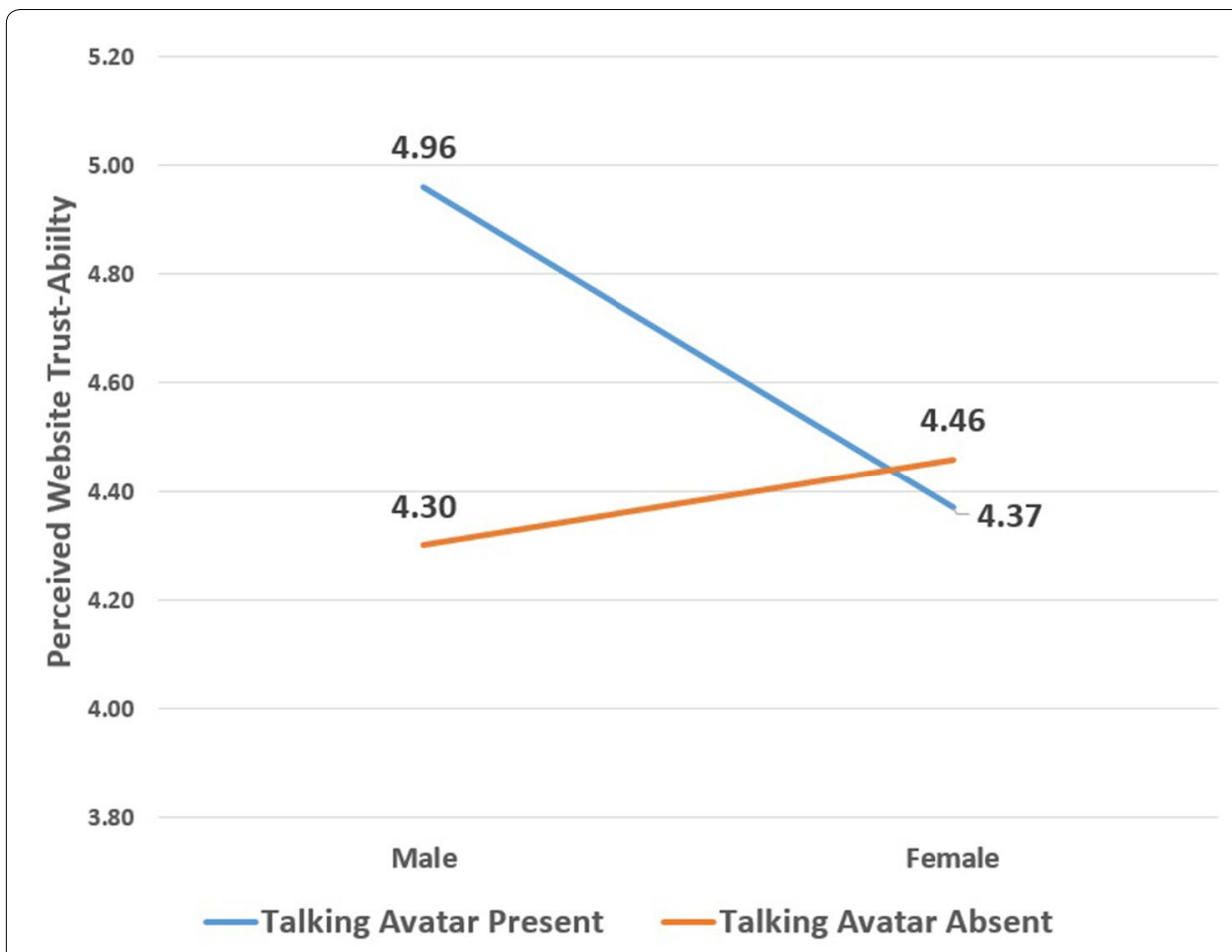

Fig. 4 Graph illustrating the interaction effect of non-interactive talking avatar presence and user's gender on website trust-integrity

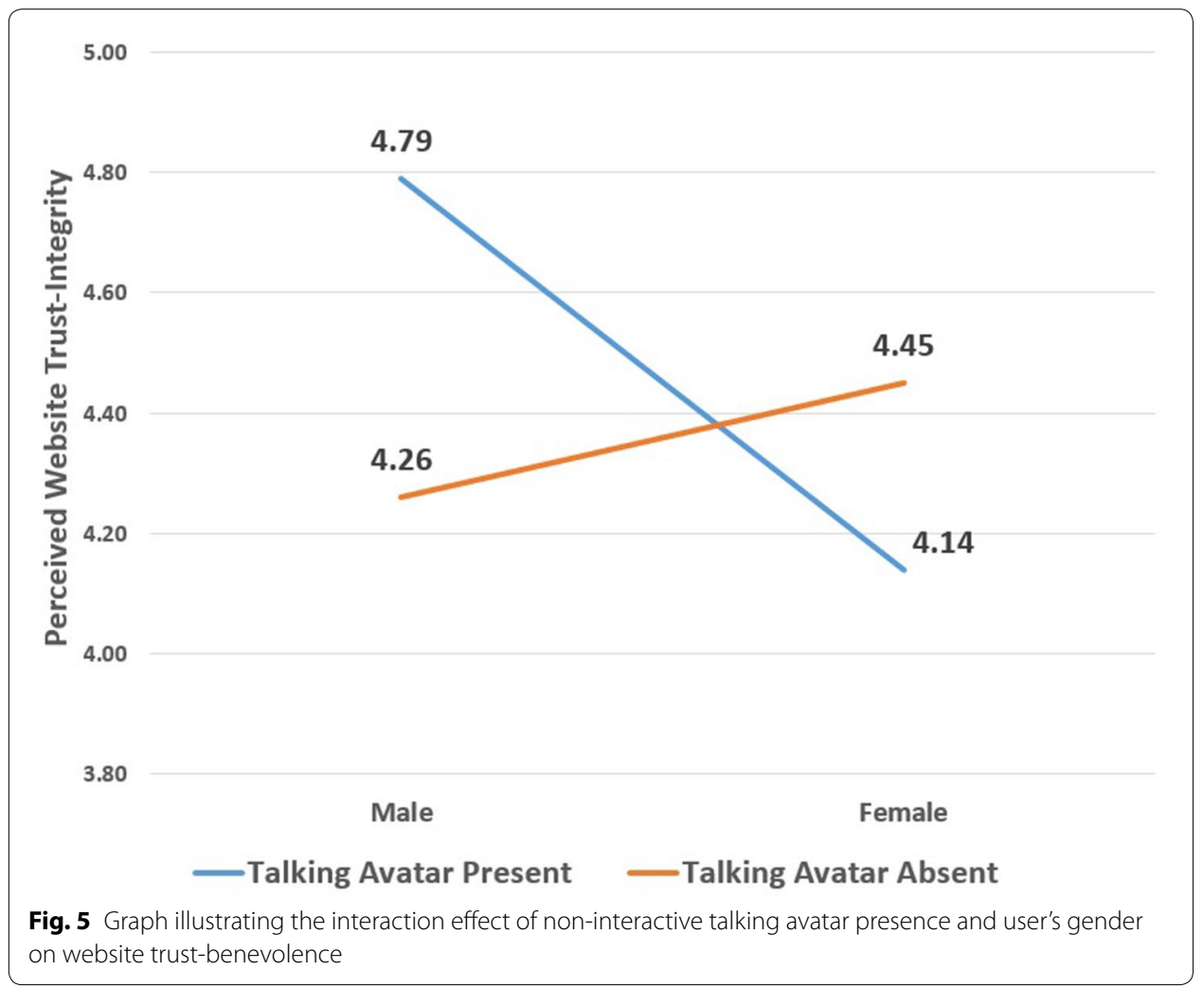




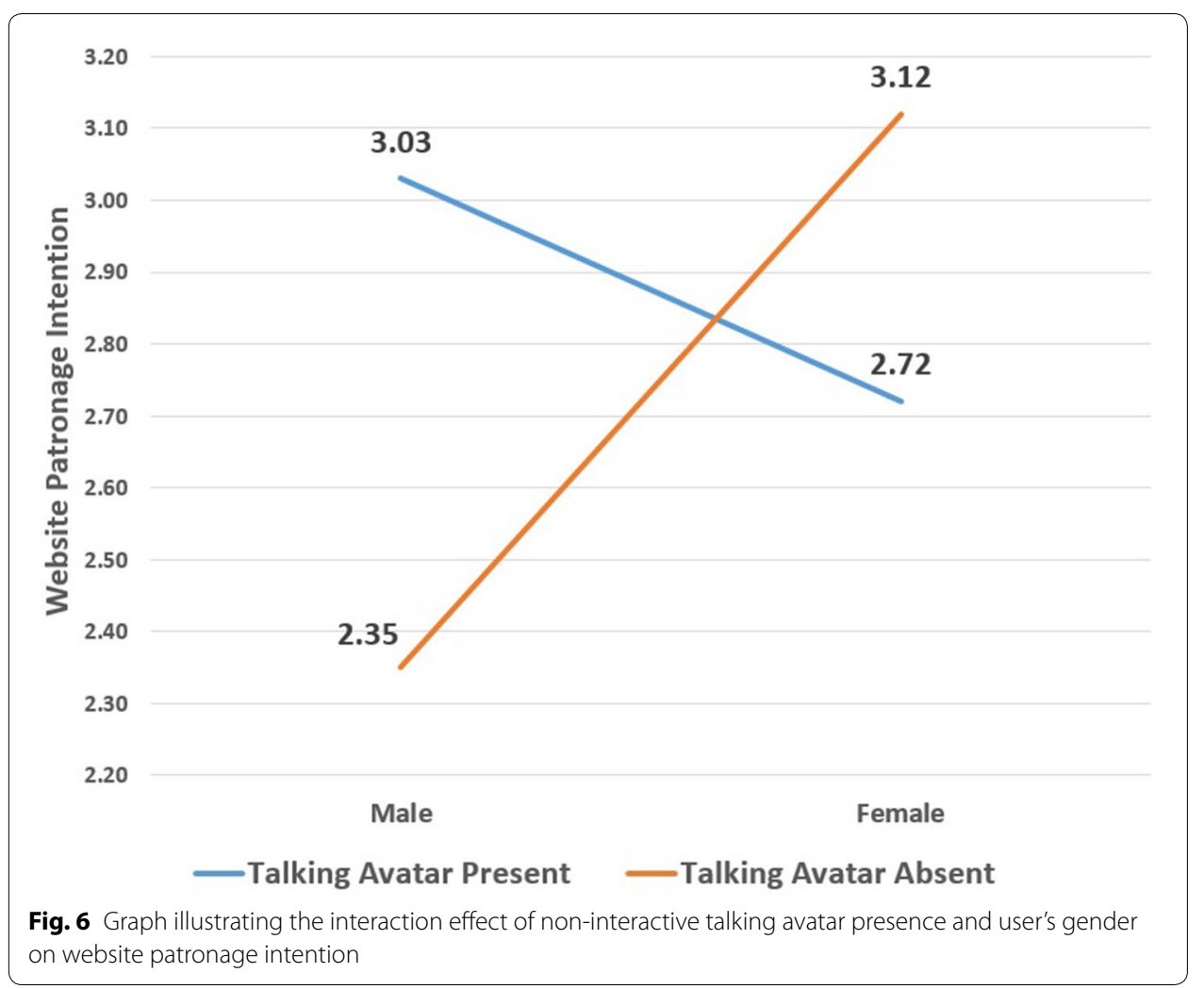

introduction of the company and products, and farewell greeting. Therefore, this finding brings fresh insights to the research domain related to e-commerce avatars, by suggesting that avatar interactivity may not be a prerequisite factor for induced social presence. Indeed, based on the social agency framework, it can be inferred that the mere visual and auditory presence of the non-interactive avatar was sufficient for simulating social schema in users' minds, thereby increasing perceptions of social presence. This observation is also in line with the Computers as Social Actors theory which argues users have the natural tendency to ascribe social agency and schemas to anthropomorphic virtual characters, especially when the objects exhibit human-like cues such as facial animation and text-to-speech voices [9].

In the context of our study, it was shown that user gender moderated the effect of non-interactive talking avatar on information credibility. Among males, perceived information credibility was neither affected by the presence nor the absence of the non-interactive talking avatar. However, it was shown that females rated the information credibility as significantly lower when it was conveyed by the talking avatar than when it was presented textually without the avatar. One plausible theoretical explanation is that the use of text-to-speech voice engendered negative perceptions of the talking avatar, which in turn, led to lower perceptions of credibility among females. Openended comments made by female users such as "I don't like the explanation about the product information because it did not explain well" and "I don't like the audio because the pronunciation is different and it takes time to understand what she says about the product" were indicative of the shortcomings of artificial sound dialogues generated by 
text-to-speech engines. Text-to-speech dialogues generally lack the quality and prosody of natural human speech, and even local accents, which may limit the perceptions of social presence in users. Earlier studies lent support to this notion [6,30]. For instance, Qiu and Benbasat [6] showed that the recommender agent with text-to-speech (without avatar image) did not invoke higher social presence than the recommender agent with only text. Moreover, it was also found that credibility was only enhanced under the presence of an avatar with human voice, whereas, the avatar with text-to-speech voice did not significantly enhance credibility when compared with the avatar-absent condition [22].

Findings from earlier works have shown that the embodied avatars with interactive capabilities can increase website trust (ability, benevolence, and integrity) in e-commerce sites $[4,5]$. In terms of website trust, our study showed that non-interactive talking avatar was only able to induce higher website trust-benevolence for both male and female users. We found interaction effects between user gender and the presence of non-interactive talking avatar on website trust-ability and website trust-integrity. Specifically, the presence of a non-interactive talking avatar increased website trust-ability and trust-integrity for male users only. Therefore, it can be inferred that non-interactive talking avatar that provides one-way greeting and product information is effective to enhance website trusts particularly for male users, by giving the impression that the website is capable and skillful to perform actions related to the business domain (ability), is sincere in placing priority on the best interest of consumers (benevolence), and is truthful and ethical in representing information, business conduct, and fulfilment of promises (integrity).

Prior works have shown that interactive avatars that simulate the roles of virtual assistants and recommender agents can enhance website patronage intentions [4, 5]. Concerning non-interactive talking avatar, our data revealed an inverse relationship between the presence of non-interactive talking avatar and user gender. Specifically, the presence of non-interactive talking avatar in the online store prompted higher patronage intention among males, while reducing patronage intention among females. This observation may be related to our previous findings on the effects of talking avatar presence on perceptions of information credibility and website trust (ability, benevolence, and integrity). Among male users, the enhanced website trust induced by the presence of a non-interactive talking avatar may have led to higher website patronage intention. In contrast, the diminished website patronage intention among females who interacted with the talking avatar may be due to negative ratings of information credibility of the talking avatar.

Our data evidently revealed that male users benefitted more from a non-interactive talking avatar than their female counterparts. Furthermore, it was shown that the presence of a non-interactive talking avatar adversely affected perceptions of information credibility and patronage intention among female participants. Prior studies have argued that females will be more susceptible to socio-emotive aspects of website design, when compared to males [3, 40]. McGoldrick et al. [21] demonstrated that females were more likely to appreciate the affable roles of talking avatars as friendly hosts than males. Following this line of reasoning, one may contend that the socio-emotive benefits of a non-interactive talking avatar would be more apparent for females than for males. However, in line with the findings of this study, it is more plausible that female participants 
who were generally more sensitive towards social cues exhibited by anthropomorphic agents $[6,19]$; had more critical impressions towards the limited interactivity of noninteractive talking avatars as compared to males. Simply put, the female participants in this study were dissatisfied and distrustful with the monologue provided by the talking Avatar. This notion was backed by remarks made by the female users such as "The information by Mina can't let me trust so much, because it didn't allow me to ask question or learn more" and "It explains each item, but it cannot reply to me", "Mina does not provide search engine, hard to find what product I want" and "Didn't have customer service like chatroom that enables consumers to contact agent/seller to know well about the product".

Moreover, the difference in information processing strategy between male and female users may also explain why the non-interactive talking avatar had a negative impact on information credibility among female users. Embodied talking avatars are agents that take the roles as proximate messengers of product information. Xiao and Tan [25] note that females are generally less trusting when receiving information from e-commerce agents than males. This is because females generally have a greater propensity for detailed and analytical processing of information, whereas, males tend to rely on heuristic information processing. On product advertisements, Papyrina [41] considers that "females would use print format as an opportunity to scrutinize ad content, whereas males would ignore it and instead resort to heuristic processing". Therefore, it can be inferred that while audio-visual information by a talking avatar appeals to heuristic information processing, the transient nature of the message evades detailed and elaborate processing of information [42]. As a result, a non-interactive talking avatar may impose on users to evaluate the message based on superficial and peripheral cues such as visual image and auditory tone. Therefore, in the context of our study, female participants might have been pressured to make heuristic and quick judgements when listening (and viewing) the product description conveyed by the non-interactive talking avatar. Some of the open-ended comments made by female participants that corroborated this notion include "It uses audio, and because of this, I am afraid that I will miss out the information about the product" and "I don't like the part where it conveys the information because I prefer to read than to hear the information about the product that it sells".

It bears mentioning that among the males, only two of the open-ended comments were about the non-interactive talking avatar; "Mina describes the product well" and "Mina provides information that can be understood". The negative comments made by the male participants revolved around the seemingly lack of product offerings; "Less products offered in catalog", "Not interesting, boring, a bit dissapointed with the categories because only got a few products" and "There are so few products for me to choose". The rest of the comments were related to the user interface of the website such as search engine and navigations; "No search engine for me to search the product. Without the search, I cannot search the product that I need to buy" and "The catalog didn't provide more pictures about the product". This observation mirrors the findings obtained in a previous study by Cyr et al. [3] which demonstrated that male consumers tend to focus on utilitarian aspects of the website such as information adequacy and security features. 


\section{Managerial implications}

Important managerial implications for e-tailers can be inferred from this study. First, it is worthwhile to recognize that embodied avatars can simulate different roles in e-commerce sites such as virtual assistants, recommender agents, and friendly hosts. Avatars that provide embodied simulacra of virtual assistants and recommender agents need to be equipped with interactive capabilities that allow real-time dialogue between users and the website. However, non-interactive avatars that simulate the roles of friendly hosts (which is the focus of this study) may be a viable option for e-tailers who want to instill a sense of social presence in their websites. With the emergence of third-party web service providers such as Sitepal and Videopal, the process of implementing non-interactive talking avatars in websites is an easy and cost-effective affair. One may argue that rather than implementing non-interactive avatars, it would be better to deploy interactive avatars. While we do not contest this notion, it is worthwhile to note that incorporating interactivity capabilities in talking avatars requires more technical expertise, time, and monetary cost as it involves the use of artificial intelligence and complex database management systems. Perhaps, this is one of the primary reasons as to why many establishments such as BobDog Wine, Qarbon Solutions, EGlobal Studies, OnCue Productions, Technology Solutions Forall, and DNA Boots utilize non-interactive talking avatars (rather than interactive avatars) in their websites.

Our study implies that the effects of non-interactive talking avatar on perceived information credibility, website trust, and patronage intention differ between male and female consumers. For female shoppers, limited interactivity and unnatural text-to-speech prosody afforded by talking avatars may decrease information credibility and patronage intention. On the other hand, website trust and patronage intention of male shoppers are enhanced through the presence of non-interactive talking avatar. Taking these observations into consideration, some managerial implications for e-tailers emerge. First, noninteractive avatars should be endowed with human voices rather than text-to-speech engines, in order to increase perceptions of information credibility, website trusts, and familiarity among female consumers. Second, as female shoppers tend to undertake a more comprehensive information processing strategy, talking avatars should only complement, rather than fully replace textual descriptions of products and services on websites. Third, e-tailers may use profiling strategy to deploy non-interactive talking avatars particularly for male and not for female shoppers. Additionally, non-interactive avatars may be incorporated in websites that offer male-gendered products (or products that are mostly bought by male shoppers), e.g., shaving creams, briefcases, and tool kits. Last but not least, e-tailers may consider sophisticated talking avatars that allow interactivity such as personalized product recommendation, intelligent search, and answers to FAQs. It should be noted, however, that the implementation of interactive avatars with human voices will cost more in terms of time, money, and effort but this may yield greater advantages in terms of information credibility, website trust, and patronage intention for both male and female online shoppers. 


\section{Limitations and avenues for future research}

Several limitations of this study warrant discussion. First, the use of university undergraduates as participants of this study might pose issues of generalizability and external validity, as this sample could not fully represent the diverse demographic qualities of online consumers. Hence, replications of this study should be conducted with different samples. Second, this study employed a controlled laboratory experiment, which provided a high level of control over the variables. Since extraneous variables were minimized, strong internal validity was assured. However, the artificial and restrictive contexts of the simulated e-commerce website, shopping task, and transitory interaction with the talking avatar, might not represent real-life scenarios of online shopping. For future studies, researchers may consider field experiments when examining the effects of non-interactive avatars in e-commerce sites. The third limitation of this study pertains to the plausible novelty effects of the talking avatar. Familiarity as a result of repeated encounters with talking avatars may potentially moderate the effects of avatars on online shopping perceptions and behaviours. Hence, in the context of this study, the short-lived talking avatar exposure might have prompted evanescent psychological and behavioural effects in participants. Further studies should employ a longitudinal design, in order to determine the replicability and generalization of the findings of this study. On a final note, talking avatar characteristics such as visual realism (stylistic versus realistic), voice (text-to-speech versus human), and non-verbal expression should be taken into account in future studies.

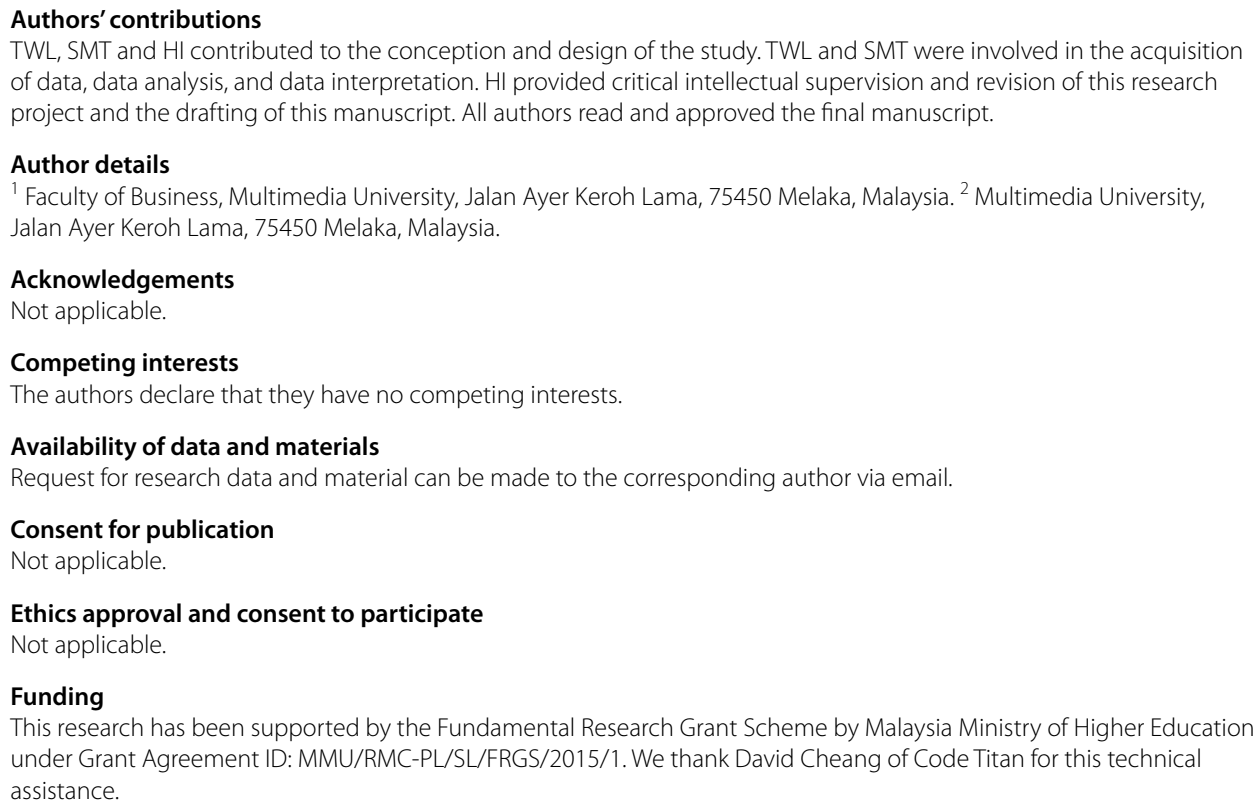

\section{Publisher's Note}

Springer Nature remains neutral with regard to jurisdictional claims in published maps and institutional affiliations. 


\section{References}

1. Gefen D, Straub D (2003) Managing user trust in B2C e-Services. e-Service J 2:7-24. https://doi.org/10.1353/ esj.2003.0011

2. Hassanein K, Head M (2007) Manipulating perceived social presence through the web interface and its impact on attitude towards online shopping. Int J Hum Comput Stud 65:689-708. https://doi.org/10.1016/j.ijhcs.2006.11.018

3. Cyr D, Hassanein $K$, Head M, Ivanov A (2007) The role of social presence in establishing loyalty in e-Service environments. Interact Comput 19:43-56. https://doi.org/10.1016/j.intcom.2006.07.010

4. Chattaraman V, Kwon W, Gilbert J (2012) Virtual agents in retail web sites: benefits of simulated social interaction for older users. Comput Hum Behav 28:2055-2066. https://doi.org/10.1016/j.chb.2012.06.009

5. Chattaraman V, Kwon W, Gilbert J, Li Y (2014) Virtual shopping agents. J Res Interact Mark 8:144-162. https://doi. org/10.1108/jrim-08-2013-0054

6. Qiu L, Benbasat I (2009) Evaluating anthropomorphic product recommendation agents: a social relationship perspective to designing information systems. J Manag Inf Syst 25:145-182. https://doi.org/10.2753/ mis0742-1222250405

7. Verhagen T, van Nes J, Feldberg F, van Dolen W (2014) Virtual customer service agents: using social presence and personalization to shape online service encounters. J Comput Med Commun 19:529-545. https://doi.org/10.1111/ jcc4.12066

8. Holzwarth M, Janiszewski C, Neumann M (2006) The influence of avatars on online consumer shopping behavior. J Mark 70:19-36. https://doi.org/10.1509/jmkg.70.4.19

9. Nass C, Brave S (2007) Wired for speech, 1st edn. MIT, Cambridge

10. Liew TW, Tan SM, Jayothisa C (2013) The effects of peer-like and expert-like pedagogical agents on learners'agent perceptions, task-related attitudes, and learning achievement. Educ Technol Soc 16(4):275-286

11. Hoyt C, Blascovich J, Swinth K (2003) Social inhibition in immersive virtual environments. Presence: Teleoperators Virtual Environ 12:183-195. https://doi.org/10.1162/105474603321640932

12. Wang $N$, Johnson W, Mayer R et al (2008) The politeness effect: pedagogical agents and learning outcomes. Int J Hum Comput Stud 66:98-112. https://doi.org/10.1016/j.ijhcs.2007.09.003

13. Liew TW, Tan SM (2016) Virtual agents with personality: adaptation of learner-agent personality in a virtual learning environment. In: IEEE digital information management (ICDIM), 2016 eleventh international conference on 2016 Sep 19, pp 157-162

14. Nass C, Lee K (2001) Does computer-synthesized speech manifest personality? Experimental tests of recognition, similarity-attraction, and consistency-attraction. J Exp Psychol Appl 7:171-181. https://doi. org/10.1037//1076-898x.7.3.171

15. Lisetti C, Amini R, Yasavur U, Rishe N (2013) I can help you change! an empathic virtual agent delivers behavior change health interventions. ACM Trans Manag Inf Syst 4:1-28. https://doi.org/10.1145/2544103

16. Liew T, Mat Zin N, Sahari N (2017) Exploring the affective, motivational and cognitive effects of pedagogical agent enthusiasm in a multimedia learning environment. Hum Centric Comput Inf Sci. https://doi.org/10.1186/ s13673-017-0089-2

17. Liew T, Zin N, Sahari N, Tan S (2016) The effects of a pedagogical agent's smiling expression on the learner's emotions and motivation in a virtual learning environment. Int Rev Res Open Distrib Learn. https://doi.org/10.19173/ irrodl.v17i5.2350

18. Kim K (2014) Can smartphones be specialists? Effects of specialization in mobile advertising. Telematics Inform 31:640-647. https://doi.org/10.1016/j.tele.2013.12.003

19. Liew T, Tan S (2017) Exploring the effects of specialist versus generalist embodied virtual agents in a multi-product category online store. Telematics and Informatics. doi:10.1016/j.tele.2017.10.005

20. Klein J, Moon Y, Picard R (2002) This computer responds to user frustration. Interact Comput 14:119-140. https://doi. org/10.1016/s0953-5438(01)00053-4

21. McGoldrick P, Keeling K, Beatty S (2008) A typology of roles for avatars in online retailing. J Mark Manag 24:433-461. https://doi.org/10.1362/026725708×306176

22. Alves A, Soares A (2013) Evaluating the use of avatars in e-commerce. Portuguese Journal of Marketing/Revista Portuguesa de Marketing 31:37-52

23. Etemad-Sajadi R (2016) The impact of online real-time interactivity on patronage intention: the use of avatars. Comput Hum Behav 61:227-232. https://doi.org/10.1016/j.chb.2016.03.045

24. Ben Mimoun M, Poncin I, Garnier M (2012) Case study_embodied virtual agents: an analysis on reasons for failure. J Retail Consumer Serv 19:605-612. https://doi.org/10.1016/j.jretconser.2012.07.006

25. Xiao BS, Tan C-W (2012) Reducing perceived deceptiveness of e-commerce product recommendation agents: an empirical examination of the relative impact of transparency and verifiability and the moderating role of gender. In: Proceedings of the eighteenth Americas conference on information systems, Seattle, Washington, August 9-12

26. Yoo Y, Alavi M (2001) Media and group cohesion: relative influences on social presence, task participation, and group consensus. MIS Quart 25:371. https://doi.org/10.2307/3250922

27. Choi Y, Miracle G, Biocca F (2001) The effects of anthropomorphic agents on advertising effectiveness and the mediating role of presence. J Interact Advert 2:19-32. https://doi.org/10.1080/15252019.2001.10722055

28. Short J, Williams E, Christie B. Visual communication and social interaction. Readings in Groupware and ComputerSupported Cooperative Work: Assisting Human-Human Collaboration, Morgan Kaufman Publishers, San Mateo, CA. 1993:153-164

29. Cyr D, Head M, Ivanov A (2009) Perceived interactivity leading to e-loyalty: development of a model for cognitiveaffective user responses. Int J Hum Comput Stud 67:850-869. https://doi.org/10.1016/j.jhcs.2009.07.004

30. Atkinson R, Mayer R, Merrill M (2005) Fostering social agency in multimedia learning: examining the impact of an animated agent's voice. Contemp Educ Psychol 30:117-139. https://doi.org/10.1016/j.cedpsych.2004.07.001

31. Beldad A, Hegner S, Hoppen J (2016) The effect of virtual sales agent (VSA) gender — product gender congruence on product advice credibility, trust in VSA and online vendor, and purchase intention. Comput Hum Behav 60:62-72. https://doi.org/10.1016/j.chb.2016.02.046 
32. Moon J, Kim E, Choi S, Sung Y (2013) Keep the social in social media: the role of social interaction in avatar-based virtual shopping. J Interact Advert 13:14-26. https://doi.org/10.1080/15252019.2013.768051

33. Koh Y, Sundar S (2010) Effects of specialization in computers, web sites, and web agents on e-commerce trust. Int J Hum Comput Stud 68:899-912. https://doi.org/10.1016/j.ijhcs.2010.08.002

34. Rousseau D, Tijoriwala S (1998) Assessing psychological contracts: issues, alternatives and measures. J Org Behav 19:679-695. https://doi.org/10.1002/(sici)1099-1379(1998)19:1

35. Harrison McKnight D, Choudhury V, Kacmar C (2002) The impact of initial consumer trust on intentions to transact with a web site: a trust building model. J Strateg Inf Syst 11:297-323. https://doi.org/10.1016/ s0963-8687(02)00020-3

36. Luhmann N (2000) Familiarity, confidence, trust: problems and alternatives. Trust Making Break Cooperat Relat 6:94-107

37. Kim J, Fiore A, Lee H (2007) Influences of online store perception, shopping enjoyment, and shopping involvement on consumer patronage behavior towards an online retailer. J Retail Consumer Serv 14:95-107. https://doi. org/10.1016/j.jretconser.2006.05.001

38. Ajzen I (1991) The theory of planned behavior. Organ Behav Hum Decis Process 50:179-211. https://doi. org/10.1016/0749-5978(91)90020-t

39. Song JH, Zinkhan GM. Features of web site design, perceptions of web site quality, and patronage behavior. In: ACME 2003 proceedings 2003 Mar 4, pp 106-114

40. Dittmar H, Long K, Meek R (2004) Buying on the internet: gender differences in on-line and conventional buying motivations. Sex Roles 50:423-444. https://doi.org/10.1023/b:sers.0000018896.35251.c7

41. Papyrina V (2013) Men and women watching and reading: gender and information processing opportunity effects in advertising. J Mark Commun 21:125-143. https://doi.org/10.1080/13527266.2012.731423

42. Chaudhuri A, Buck R (1995) Media differences in rational and emotional responses to advertising. J Broadcast Electr Media 39:109-125. https://doi.org/10.1080/08838159509364291

\section{Submit your manuscript to a SpringerOpen ${ }^{\circ}$ journal and benefit from:}

- Convenient online submission

Rigorous peer review

- Open access: articles freely available online

- High visibility within the field

Retaining the copyright to your article

Submit your next manuscript at $\mathbf{s p r i n g e r o p e n . c o m ~}$ 